\section{Asthma bronchiale: SMART-Konzept reduziert Exazerbationsrate}

Sobireraj DM et al. Association of Inhaled Corticosteroids and Long-Acting $\beta$-Agonists as Controller and Quick Relief Therapy With Exacerbations and Symptom Control in Persistent Asthma. JAMA 2018; 319: 1485 - 1496

Bei einem nicht kontrollierten Asthma bronchiale empfehlen Leitlinien die stufenweise Steigerung der inhalativen Kortikosteroide und die $\mathrm{Er}$ gänzung mit langwirksamen $\beta$-Agonisten. Als schnelle Atemhilfe stehen kurzwirksame $\beta$-Agonisten zur Verfügung. Die Metaanalyse stellte nun Studien zusammen, die die Überlegenheit eines Kombinationsinhalators nicht nur für die Controller, sondern auch als Reliever nahelegen.

Internationale Leitlinien empfehlen das SMART-Konzept (Single Maintainance and Reliever Therapy), d. h. die Kombination inhalativer Kortikosteroide (ICS) und langwirksamer $\beta$-Agonisten (LABA) für beide Szenarien: für die dauerhafte Kontrolle des mittelschweren und schweren Asthma bronchiale sowie für den akuten Bedarfsfall. Der schnelle Wirkungseintritt des LABA Formoterol spreche für die Nutzung über die Dauermedikation hinaus. Für den dualen Ansatz bestehe in den USA aber bislang keine Zulassung, so die Autoren.

Sie fanden insgesamt 16 randomisierte Studien mit 22748 Patienten, die den Nutzen der SMART-Strategie mit den Standardmedikationen verglichen. In 15 Studien verwandten die Wissenschaftler Pulverinhalatoren mit Budenosid/Formoterol. Das SMART-Konzept wurde mit einer Dauermedikation aus ICS oder ICS/ LABA verglichen. Zusätzlich konnten akut kurzwirksame $\beta$-Agonisten benutzt werden (SABA). Bei Patienten $\geq 12$ Jahren war die SMART-Strategie mit signifikant weniger schweren Exazerbationen assoziiert, die ein Kompositum aus Hospitalisation, Notfallbehandlung und systemischen Kortikosteroiden definierte. Verglichen mit ICS/LABA in gleicher Dosierung plus SABA im Bedarfsfall war SMART mit einem geringeren Risiko für Exazerbationen assoziiert (Relatives Risiko 0,68; 95\%-Konfidenzintervall $\quad 0,58-$ $0,80)$. Aus der Verwendung der Fixkombination auch im Bedarfsfall resultierte eine höhere ICS- und LABA-Gesamtdosis. SMART beeinflusste leichte Exazerbationen nicht. Verglichen mit den Standardmedikationen bestand kein besonderer Zusammenhang mit spirometrischen Daten als Kriterien für die Krankheitskontrolle (funktionelle Einsekundenkapazität FEV1, funktionelle Vitalkapazität FVC). Asthma-spezifische Todesfälle kamen in den Studien nicht vor. Nur 1 Studie beobachtete Kinder von 4-11 Jahren, für die ebenfalls eine reduzierte Exazerbationsrate mit SMART vermutet wurde. Die Lebensqualität war selten Untersuchungsgegenstand. SMART beeinflusste in diesen Untersuchungen die Asthma-assoziierte Lebensqualität nicht anders als der Standard.

\section{FAZIT}

Verglichen mit allen Komparatoren reduzierte die SMART-Strategie die Häufigkeit ernsthafter Exazerbationen bei Patienten mit mäßigem und schwerem Asthma bronchiale. Auf die Kontrolle des Asthmas insgesamt und die Lebensqualität wirkte sich das Konzept nicht zusätzlich aus. Der höheren inhalativen Kortikosteroiddosis stellen die Autoren den reduzierten Gebrauch systemischer Kortikosteroide gegenüber.

Dr. med. Susanne Krome, Melle 